\title{
HDEEP: Heterogeneous Distributed Energy Efficient Protocol for Clustered WSNs
}

\author{
K. M. Gazala \\ Department of computer \\ science and engineering \\ Sharda University, \\ Greater Noida,India
}

\author{
Sudeep Varshney \\ Department of computer \\ science and engineering \\ Sharda University, \\ Greater Noida,India
}

\begin{abstract}
Wireless sensor network (WSN) is widely accepted as a standout amongst the most fundamental advancements for the twenty-first century. In the previous decades, it has agreed inconceivable consideration from both the scholarly world and industry all over the world. A WSN typically comprises of countless cost, low-control, and multifunctional remote sensor nodes, close by recognizing, remote contact and calculation capacities. Current advances in micro-electromechanical arrangements (MEMS) knowledge, wireless sensors, and digital electronics have enabled the progress of low-cost, low-power, multifunctional sensor nodes that are tiny in size and converse unmetered in short distances. These puny sensor nodes, that encompass of detecting, data processing, and conversing constituents, impact the believed of sensor networks established on cooperative power of a colossal number of nodes.
\end{abstract}

\section{Keywords}

Wireless sensor network, Energy Efficiency, LEACH, EEM LEACH, Clustering.

\section{INTRODUCTION}

Wireless sensor network (WSN) is extensively believed as one of the most vital technologies for the twenty-first century. The word "wireless" has come to be a generic and allencompassing word utilized to delineate contact in that electromagnetic waves are utilized to hold a gesture above portion or the whole contact path. Wireless knowledge can able to grasp nearly every single locale on the external of the earth. Due to incredible accomplishment of wireless voice and messaging services, it is hardly stunning that wireless contact is commencing to be requested to the area of confidential and company computing. Ad- hoc and Sensor Networks are one of the portions of the wireless communication. In ad-hoc web every single and every single nodes are permit to converse alongside every single supplementary lacking each fixed infrastructure. This is truly one of the features that differentiate amid ad-hoc and supplementary wireless knowledge like cellular webs and wireless LAN that truly needed ground work established contact like across a little center station.

"A sensor network is an arrangement of huge amount of tiny, inexpensive, self-powered mechanisms that is used to sense, calculate, and converse alongside supplementary mechanisms for the intention of meeting innate data to make globe decisions concerning a physical environment." Sensor networks embody a momentous enhancement above established sensors, that are used in the pursuing two ways:
- Sensors can be situated at an awesome separation from the real wonder, i.e., something known by sense discernment. In this approach, expansive sensors that utilization some intricate way to deal with investigate the objectives from natural commotion are required.

- Several sensors that perform no one but detecting can be sent. The positions of the sensors and correspondences topology are deliberately designed. They transmit time arrangement of the detected wonder to the focal hubs where calculations are performed and information are combined.

\section{RELATED WORK}

Wendi(Wendi et al 2000)They presented LEACH as a clustering based protocol and also do it utilizes irregular movement of regional cluster base stations to equally dispersed energy load among the sensors in the wireless sensor network. They simulated the network that proved LEACH is capable of dispersion energy dissipation equally throughput the sensors ,that helps to Double the useful system lifetime

Jiguo(Jiguo Yu et al 2008) a multi hop routing protocol that is energy efficient (EEMR) is proposed for WSN. It mainly focused on multi hop route. This algorithm says that cluster head that is far from the BS has the bigger cluster size but $\mathrm{CH}$ that is near to BS has the smaller $\mathrm{CH}$ size. This algorithm is helpful for enhancement of network lifetime.

Shio(Shio et al 2010) In this paper they driven the primary concentration on the study of the cluster based hierarchical energy efficient at hand routing for WSN. They have done study to primarily focus on the cluster based hierarchical energy efficient protocol for WSN. This paper covers only some samples of routing protocol, as WSN is very wide area.

Jong (Jong-Shinchen et al 2010) in this paper, they classified the lifetime into various kinds and given the equivalent $\mathrm{CH}$ selection technique to execute the lifetime expansion objective .Their study enlarged the lifetime for various requests of the sensor networks. They proposed effective request oriented coordinator techniques for hierarchical sensor networks.

Coverage of nodes is an essential task in WSN. To improve coverage in WSN (Zhixin et al 2012) has given a algorithm DEECIC that has goal to cover the entire network via less amount of cluster heads. This algorithm also enhance network lifetime and gives improvement on coverage of network effectively. 
Ahmad (Ahmad et al 2013) This Research paper presented a very famous routing method based on clusters in Wireless Sensor Networks (WSNs). Because WSN applications need continuously changing, there are many possible field of research on how energy can be efficiently used in routing protocols. This paper is very useful to solve the difficulty of energy hole and coverage hole.

Nikolaos (Nikolaos A. Pantazis et al 2013) In this paper types of energy efficient routing protocol is described. They are of four types- Communication model, Reliable routing, Network structure and topology based. A rational study on energy efficient routing protocol for WSNs is given. In this paper they mainly focused on the energy efficient protocols designed for Wireless Sensor Network.

Rodrigo (Rodrigo S. Semente et al., 2013 ) In this work, a Wireless Sensor Networks (WSN) is analyzed and implemented to control a instrumented process in environments with limited power source. Present propose is based on IEEE 802.15.4 standard, using Zigbee and Modbus protocols.

Raed (Raed Bani-Hani 2013) In this paper they presented a study of LEACH, that is a hierarchical routing protocol and also they explained how energy consumption can be improved in WSN and how can increase network's lifetime. Because of some defects of LEACH, Several methods are combined to solve the difficulties. Although to find better efficient scalable and, robust clustering protocols some work is still required that can increase energy consumption and prolong network lifetime in tiny as well as large WSN.

Antoo(Antoo Ashlyn et al, 2014) This paper proposes a energy proficient directing convention EEM-LEACH that finds a multihop way with least correspondence cost from every node to the base station. In the event that the correspondence cost for direct information exchange is least, nodes near the base station dispatch information to the base station directly in this way keeping them from dying soon. Only nodes with maximum residual energy and minimum energy consumption can become cluster heads since each node's residual energy as well as average energy consumption is considered for the selection of cluster heads. No global information is needed because cluster formation and multi-hop path selection are distributed.

\section{HETEROGENOUS ENERGY EFFICIENT ROUTING PROTOCOL (HDEEP)}

Proposed routing protocol was developed by introducing the feature of energy aware routing and multi-hop intra cluster routing. The operation of the Proposed protocol is separated into rounds where each round starts with a set-up stage, when the groups are composed, trailed by a steady state stage, when information exchanges to the base station happen. The below flow chart describes the overview of the protocol, initially the user has to give input in the form of rounds.

For the nodes produced, their positions are randomly appointed and showed. Once the nodes are deployed, each node uses the neighbor discovery algorithm to find its neighbor nodes. Utilizing the cluster head choice calculation cluster heads are chosen among the nodes. These cluster heads telecasts the advertisement message to all its neighboring nodes and thus clusters are shaped with a fixed bound size. Every node in the cluster keeps up directing table in which routing information of the nodes are upgraded Distributed randomized time slot assignment algorithm strategy is utilized, it permits a few nodes to have the same frequency channel by separating the signal into various time slots. The cluster head totals the information from every one of the nodes in the cluster and this collected information is transmitted to the base station. Once the clusters are made, the sensor nodes are allotted timeslots to send the information. Assuming nodes always have information to send, they transmit it at their designated time interval.

When node gets information from one its neighbors, it totals it with its own particular information. While sending the collected information, it needs to pick an ideal way from its routing table sections. It utilizes a heuristic function to make this decision and the heuristic function is given by,

$\mathrm{h}=\mathrm{K}(\mathrm{E}$ avg/hmin $)$

where $\mathrm{K}$ is a consistent, Eavg is average energy of the current path, hmin is minimum hop count in current path, $\mathrm{t}=$ traffic in the current path. The path with highest heuristic value is picked. If this path's Emin> threshold, it is picked. Else the path with the next highest heuristic value is picked, where Emin $=$ Eavg/const

The below flowchart describes the mechanism of the proposed protocol HDEEP. The working of the protocol is divided into phases:

1. Set up phase

2. Data transmission phase 


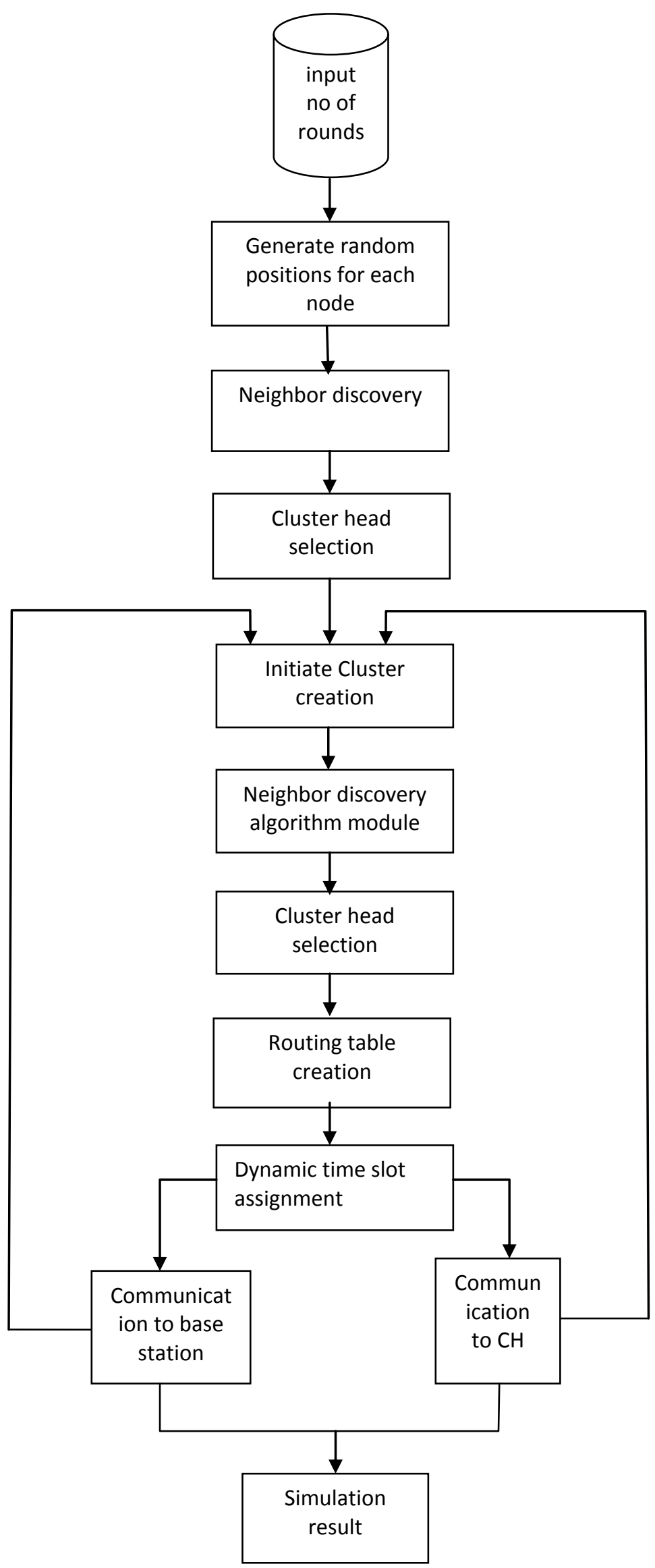

Fig 1:Flowchart of proposed Model 


\subsection{Setup Phase}

At first, after the nodes arrangement the neighbor discovery happens. This can be possible utilizing numerous techniques like: k-of-n methodology, ping, beacon messaging. After the neighbor discovery, when clusters are being made, every node chooses whether or not to wind up a cluster head for the current round. This choice strategy is like the one utilized as a part of LEACH. The setup stage works in the following sequence:

\subsubsection{Cluster Head Selection and Cluster Formation}

Clusters are formed utilizing a distributed algorithm here the thought is for the sensor nodes to choose themselves as for their energy levels self-sufficiently. The objective is to minimize communication cost and maximizing network resources to guarantee that concise data is sent to the sink. Every node transmits information to the nearest cluster head and the cluster heads perform data aggregation. We continue to our indicator function of picking a cluster head. Assume an ideal number of clusters $\mathrm{k}$ in each round. It is normal that as a cluster head, more energy will be consumed than being a cluster member. Every node can get to be cluster head with a probability $\square$ opt and each node must get to be cluster head once every ( $1 / \square$ opt) rounds. Instinctively, it implies we have ( $\square \cdot \square$ opt) clusters and cluster heads per round. Let the non-elected nodes be a member of set $\mathrm{G}$ in the past ( $1 / \square \mathrm{opt}$ ) rounds.

\subsubsection{Heterogeneous WSN Model}

We assume $\mathrm{N}$ number of nodes set in a square area of measurement $\mathrm{M} \times \mathrm{M}$. Heterogeneous WSNs contain two, three or multi types of nodes as with respect to their energy levels and are termed as two, three and multi level heterogeneous WSNs individually.

\subsubsection{Two Level Heterogeneous WSNs Model}

Two level heterogeneous WSNs contain two energy level of nodes, normal and advanced nodes. Where, Eo is the energy level of normal node and $\operatorname{Eo}(1+$ an $)$ is the energy level of advanced nodes containing a times more energy when contrasted with normal nodes. In the event that $\mathrm{N}$ is the aggregate number of nodes then $\mathrm{Nm}$ is the number of advanced nodes where $m$ refers to the division of advanced nodes and $\mathrm{N}(1-\mathrm{m})$ is the quantity of normal nodes. The total initial energy of the system is the whole of energies of normal and advanced nodes.

Etotal $=\mathrm{N}(1-\mathrm{m})$ Eo $+\mathrm{Nm}(1+\mathrm{a})$ Eo

$=\mathrm{NEo}(1-\mathrm{m}+\mathrm{m}+\mathrm{am})$

$=\mathrm{NEO}(1+\mathrm{am})$

The two level heterogeneous WSN contain am times more energy when contrasted with homogeneous WSNs.

\subsubsection{Three Level Heterogeneous WSN Model}

Three level heterogeneous WSNs contain 3 entirely unexpected energy levels of nodes i.e normal, advanced and super hubs. Normal nodes contain energy of Eo, the advanced nodes of part $\mathrm{m}$ territory unit having a times extra energy than normal nodes satisfactory $\operatorname{Eo}(1+a)$ while, super nodes of division mo zone unit having a component of $\mathrm{b}$ times a considerable measure of energy than normal nodes along these lines their energy is sufficient $\operatorname{Eo}(1+b)$. As $\mathrm{N}$ is that the aggregate scope of nodes inside the system, then Nmmo is all out scope of super nodes and $\mathrm{Nm}(1-\mathrm{mo})$ is total number of advanced nodes. the whole introductory energy of 3 level heterogeneous WSN is so given by:

Etotal $=\mathrm{N}(1-\mathrm{m}) \mathrm{Eo}+\mathrm{Nm}(1-\mathrm{mo})(1+\mathrm{a}) \mathrm{Eo}+\mathrm{NmoEo}(1+$ b)

$($ Etotal $=\mathrm{NEo}(1+\mathrm{m}(\mathrm{a}+\mathrm{mob}))$

The three level heterogeneous WSNs contain $(\mathrm{a}+\mathrm{mob})$ times more energy as compared to homogeneous WSNs

\subsubsection{Multilevel Heterogeneous WSN Model}

Multi level heterogeneous WSN is a network that contains nodes of multiple energy levels. The initial energy of nodes is distributed over the close set [Eo,Eo(1+amax $)]$, where Eo is the lower bound and amax is the value of maximal energy. Initially, node $\mathrm{Si}$ is equipped with initial energy of $\mathrm{Eo}(1+\mathrm{ai})$, which is ai times more energy than the lower bound Eo. The total initial energy of multi-level heterogeneous networks is given by:

$$
E_{\text {total }}=\sum_{i=1}^{N} E_{0}\left(1+a_{i}\right)=E_{0}\left(N+\sum_{i=1}^{N} a_{i}\right)
$$

$\mathrm{CH}$ nodes consume more energy as compared to member nodes so after some rounds energy level of all the nodes becomes different as compared to each other. Therefore, heterogeneity is introduced in homogeneous WSNs and the networks that contain heterogeneity are more important than homogeneous networks.

\subsection{Data Transmission Phase}

Once the clusters are created, the sensor nodes are allotted timeslots to send the data. Assuming nodes always have data to send, they transmit it at their allotted time interval. When a node receives data from one its neighbors, it aggregates it with its own data. While forwarding the aggregated data, it has to choose an optimal path from its routing table entries. It uses a heuristic function to make this decision and the heuristic function is given by,

$\mathrm{h}=\mathrm{K}(\mathrm{E}$ avg/h $\min )$

where $\mathrm{K}$ is a constant, Eavg is average energy of the current path, $\mathrm{h}$ min is minimum hop count in current path, $\mathrm{t}=$ traffic in the current path.

The path with highest heuristic value is chosen. If this path's Emin> threshold, it is chosen. Else the path with the next highest heuristic value is chosen, where

Emin $=$ Eavg $/$ const

The constant may be any integer value like 10

If no node in the routing table has Emin greater than threshold energy, it picks the node with highest minimum energy.

\section{SIMULATION AND ANALYSIS OF RESULT}

Both LEACH and Proposed protocol are simulated using MATLAB. The parameters taken into consideration while evaluating Proposed protocol and LEACH are as follows. 
- $\quad$ Round Number vs. Number of Dead Nodes (with variation of probability)

- $\quad$ Round Number vs. Average Energy of Alive nodes (with variation of probability)

- Round Number vs. Number of Alive Nodes (with variation of number of nodes)

- $\quad$ Round Number vs. Data packet delivery of nodes (With variation of number of nodes)

- To simplify the simulation of these protocols few assumptions are made. They are as follows:

- Initial energy of nodes is same.

- $\quad$ Nodes are static

- Nodes are assumed to have a limited transmission range after which a another equation for energy dissipation is used

- Homogeneous distribution of nodes.

- Nodes always have to send the data.

Details of the simulation Parameters are mentioned in Table 1, given below:

The selection of energy efficient routing protocol depends on the applications. Thus, it is necessary to compare the results of different energy efficient routing techniques.

In order to evaluate the performance of proposed technique, a MATLAB simulator has been used. Table 1 contains the standard parameters of environmental parameters used in the simulation.

Table1: Simulation Parameters

\begin{tabular}{|c|c|}
\hline Parameters & Values \\
\hline WSN Area & $100 \mathrm{~m} \mathrm{x} \mathrm{100m}$ \\
\hline Base Station Location & $50 \mathrm{~m}, 50 \mathrm{~m}$ \\
\hline Number of sensor nodes & 100 nodes \\
\hline Initial node energy & $0.1 \mathrm{~J}$ \\
\hline Percentage of CH selection & 0.05 \\
\hline $\begin{array}{c}\text { Transmitter/Receiver } \\
\text { electronics } \mathrm{E}_{\text {elec }}\end{array}$ & $50 \mathrm{~nJ} / \mathrm{bit}$ \\
\hline $\begin{array}{c}\text { Transmitter amplifier } €_{\mathrm{fs}} \\
\left(\mathrm{d}<\mathrm{d}_{0}\right)\end{array}$ & $10 \mathrm{pJ} / \mathrm{bit} / \mathrm{m}^{2}$ \\
\hline $\begin{array}{c}\text { Transmitter amplifier } \\
€_{\mathrm{mp}}\left(\mathrm{d}>\mathrm{d}_{0}\right)\end{array}$ & $0.0013 \mathrm{pJ} / \mathrm{bit} / \mathrm{m}^{4}$ \\
\hline $\begin{array}{c}\text { The energy for aggregation } \\
\mathrm{E}_{\mathrm{DA}}\end{array}$ & $5 \mathrm{n} \mathrm{J} / \mathrm{bit} / \mathrm{signal}$ \\
\hline
\end{tabular}

The determination of energy effective directing protocol relies on upon the applications. In this way, it is important to think about the aftereffects of various energy productive steering procedures. To assess and think about the execution of these conventions, 2 execution measurements are utilized:
1. Network Lifetime: Number of live nodes in each round

2. Data Packet Delivery: Data packets conveyed to the base station in each round.

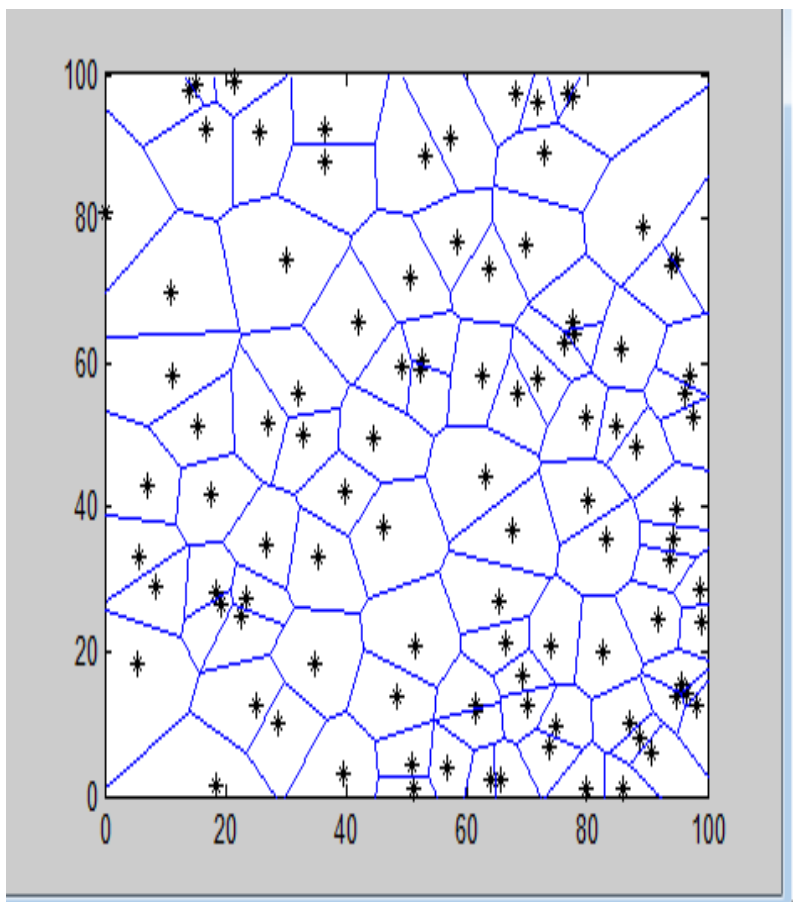

Fig2 : Wireless sensor network over an area of $100 \mathrm{~m} x$ $100 \mathrm{~m}$

A wireless sensor network consisting of 100 nodes are distributed randomly over an area of $100 \mathrm{~m} \times 100 \mathrm{~m}$ and is given in Fig. 2.Sink is located at the centre $(50 \mathrm{~m}, 50 \mathrm{~m})$

The proposed protocol when compared with the EEM LEACH protocol,the proposed protocol increases the network lifetime of the protocol.Table2 shows the comparison of network lifetime of EEM LEACH protocol and the proposed HDEEP protocol.

Table 2:Comparison Of Network Lifetime Of EemLeach And Hdeep

\begin{tabular}{|c|c|c|c|}
\hline Network & Protocol & $\begin{array}{c}\text { First } \\
\text { node } \\
\text { dead }\end{array}$ & $\begin{array}{c}\text { Last } \\
\text { node } \\
\text { dead }\end{array}$ \\
\hline $\begin{array}{c}100 \mathrm{~m} \\
\mathrm{x} 100 \mathrm{~m}\end{array}$ & $\begin{array}{c}\text { EEM } \\
\text { LEACH }\end{array}$ & 205 & 247 \\
\cline { 2 - 4 } & HDEEP & 387 & 879 \\
\hline
\end{tabular}




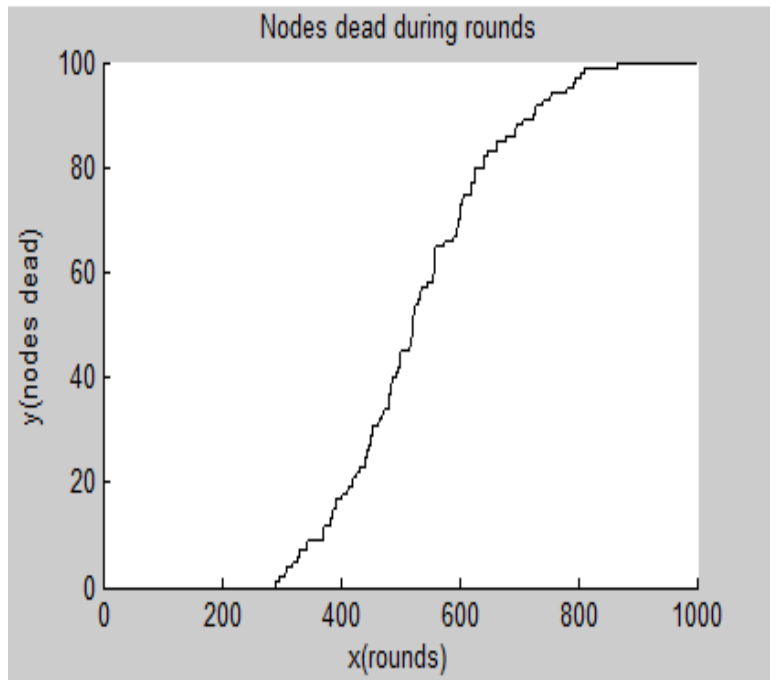

Fig 3: Nodes dead over an area of $100 \mathrm{~m} \times 100 \mathrm{~m}$ area network

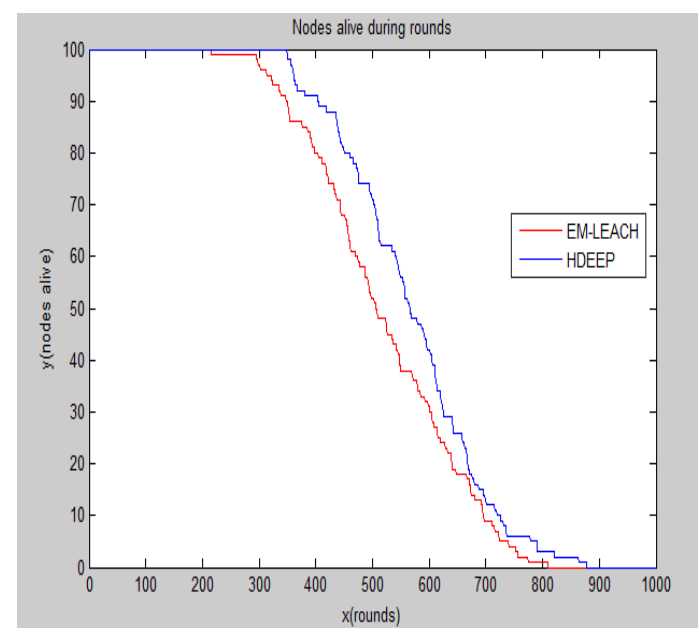

Fig 4: Comparison of network lifetime of EEM LEACH and HDEEP

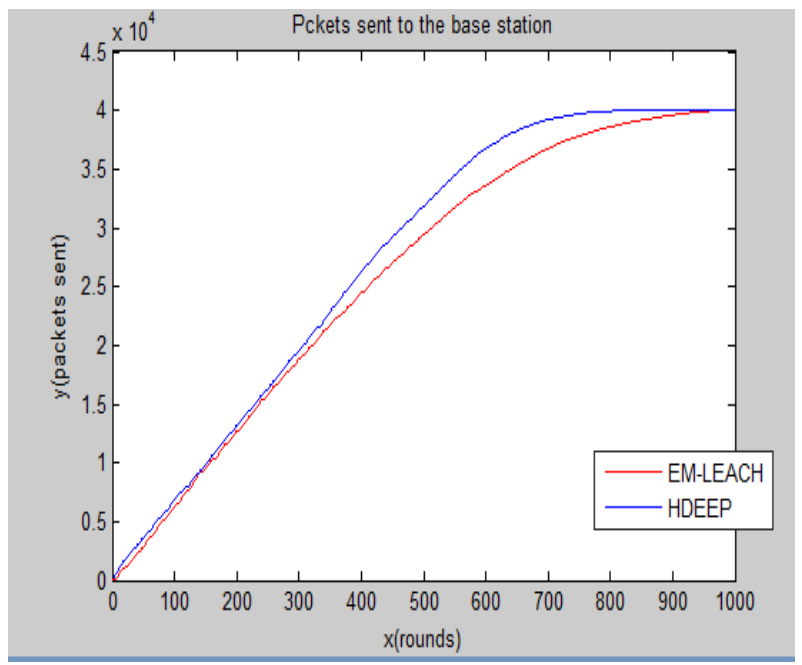

Fig 5 : Data packet delivery to the base station

\section{CONCLUSION}

In our work we have studied the impact of heterogeneity of nodes, in terms of their energy, in wireless sensor networks that are hierarchically clustered. In these networks some of the nodes become cluster heads, aggregate the data of their cluster members and transmit it to the sink. It is assumed that a percentage of the population of sensor nodes is equipped with additional energy resources - this is a source of heterogeneity which may result from the initial setting or as the operation of the network evolves.

Classical clustering protocols assume that all the nodes are equipped with the same amount of energy and as a result, they can not take full advantage of the presence of node heterogeneity. we proposed, a heterogeneous-aware protocol to prolong the time interval before the death of the first node, which is crucial for many applications where the feedback from the sensor network must be reliable. The proposed work greatly improved the Energy Efficiency and Overall stability of the Heterogeneous WSN Network.

\section{REFERENCES}

[1] Lavratti, F., Pinto, A.R., Bolzani, L., Vargas, F., Montez, C., Hernandez, F., Gatti, E., Silva, C., "Evaluating a Transmission Power Self-Optimization Technique for WSN in EMI Environments", PUCRS, Catholic Univ. of Rio Grande do Sul, Porto Alegre, Brazil, 10.1109/DSD.2010.116, 509-515, 2010

[2] Teubler, T., Hail, M.A., Hellbruck, H., "Transparent Integration of Non-IP WSN into IP Based Networks", Electr. Eng. \&Comput. Sci., Lubeck Univ. of Appl. Sci., Lubeck, Germany, 10.1109/DCOSS.2012.10, 353-358, 2012

[3] Alaiad, A., Lina Zhou, "Patients' Behavioral Intentions toward Using WSN Based Smart Home Healthcare Systems: An Empirical Investigation", Dept. of Inf. Syst., Univ. of Maryland-Baltimore County, Baltimore, MD, USA 10.1109/HICSS.2015.104, 824-833, 2015

[4] Aslam, M.S., Rea, S., Pesch, D., "Service Provisioning for the WSN Cloud", Nimbus Centre For Embedded Syst. Res., Cork Inst. of Technol., Cork, Ireland, 10.1109/CLOUD.2012.132, 962-969, 2012

[5] Bellavista, P., Cardone, G., Corradi, A., Foschini, L., "Convergence of MANET and WSN in IoT Urban Scenarios", Dept. of Comput. Sci. \& Eng., Univ. of Bologna, Bologna, Italy, 10.1109/JSEN.2013.2272099, 3558-3567, 2013

[6] Cardone, G., Bellavista, P., Corradi, A., Foschini, L., "Effective collaborative monitoring in smart cities: Converging MANET and WSN for fast data collection", Dipt. di Elettron., Inf. e Sist., Univ. of Bologna, Bologna, Italy, , 1-8, 2011

[7] Rajgarhia, P., James, P., Nainwal, V., Sowjanya, P., Koshy, S.S., "Development of an Ultra-low power WSN gateway for outdoor deployments", Embedded Syst. \& Design Team, Centre for Dev. of Adv. Comput. (C-DAC), Hyderabad, India, 10.1109/ICICES.2014.7034151, 1-7, 2014

[8] Gaillard, G., Barthel, D., Theoleyre, F., Valois, F., "Service Level Agreements for Wireless Sensor Networks: A WSN operator's point of view", Orange Labs. R\&D, Meylan, France, 10.1109/NOMS.2014.6838261, 1-8, 2014 
[9] Marchiori, A., Qi Han, "PIM-WSN: Efficient multicast for IPv6 wireless sensor networks", Dept. of Math. \&Comput. Sci., Colorado Sch. of Mines, Golden, $\mathrm{CO}$,

USA, 10.1109/WoWMoM.2011.5986469, 1-6, 2011

[10] Rea, S., Aslam, M.S., Pesch, D., "Serviceware - A service based management approach for WSN cloud infrastructures", Nimbus Centre for Embedded Syst. Res., Cork Inst. of Technol., Cork, Ireland, 10.1109/PerComW.2013.6529470, 133-138, 2013

[11] Jun Lei, WenjiaNiu, Yifang Qin, Hui Tang, Song Ci, "Aggregating user rating and service context for WSN service ranking", High Performance Network Lab., Inst. of Acoust., Beijing, China, 10.1109/GLOCOM.2012.6503973, 5362-5367, 2012

[12] Raza, F., Bashir, S., Tauseef, K., Shah, S.I., "Optimizing nodes proportion for intrusion detection in uniform and Gaussian distributed heterogeneous
WSN", Dept. of Telecom \& Networks, Iqra Univ., Islamabad,

Pakistan,

10.1109/IBCAST.2015.7058571, 623-628, 2015

[13] ZhoubingXiong, Sottile, F., Spirito, M.A., Garello, R., "Hybrid Indoor Positioning Approaches Based on WSN and RFID", Politec. di Torino, Turin, Italy, 10.1109/NTMS.2011.5721059, 1-5, 2011

[14] Merentitis, A., Kranitis, N., Paschalis, A., Gizopoulos, D., "Low Energy Online Self-Test of Embedded Processors in Dependable WSN Nodes", Dept. of Inf. \&Telecommun., Nat. \&Kapodistrian Univ. of Athens, Athens, Greece, 10.1109/TDSC.2011.17, 86-100, 2012

[15] Serdaroglu, K.C., Baydere, S., "Seamless interconnection of WSN and internet", Dept. of Comput. Eng., Yeditepe Univ., Istanbul, Turkey, , 16, 2012 